\title{
Regulation of p38 MAPK phosphorylation inhibits chondrocyte apoptosis in response to heat stress or mechanical stress
}

\author{
KEN TAKEBE, TAKAYUKI NISHIYAMA, SHINYA HAYASHI, SHINGO HASHIMOTO, \\ TAKAAKI FUJISHIRO, NORIYUKI KANZAKI, KOHEI KAWAKITA, KENJIRO IWASA, \\ RYOSUKE KURODA and MASAHIRO KUROSAKA
}

\author{
Department of Orthopaedic Surgery, Kobe University Graduate School of Medicine, Kobe, Hyogo 670-0017, Japan
}

Received October 4, 2010; Accepted November 29, 2010

DOI: $10.3892 /$ ijmm.2010.588

\begin{abstract}
Activation of p38 MAPK has been associated with a stress response and with apoptotic processes. However, the function of p38 MAPK in chondrocytes is not clearly understood. In this study, we analyzed the expression of p38 MAPK in chondrocytes and investigated the function of p38 MAPK in response to heat stress and mechanical stress. Chondrocytes were isolated from human cartilage and cultured. Expression of p38 and phosphorylated p38 in cartilage of patients with osteoarthritis (OA) was compared to those in normal cartilage by immunohistochemistry and Western blotting. Human knee chondrocytes were exposed to heat stress or mechanical stress. Normal knee chondrocytes were pre-treated with SB203580 or p38 small interfering RNA (siRNA) before induction of heat stress or mechanical stress. Chondrocyte apoptosis was detected by TUNEL staining and Western blotting of cleaved caspases. OA and normal chondrocytes expressed p38; however, OA chondrocytes showed much higher phosphorylated p38 compared to normal chondrocytes. Heat stress or mechanical stress induced apoptosis and increased phosphorylated p38 in normal chondrocytes. The TUNEL positive cells and expression levels of phosphorylated p38 in response to stress decreased when chondrocytes were incubated with SB203580 or transfected with siRNA against p38. In conclusion, we have demonstrated that heat stress or mechanical stress increased chondrocyte apoptosis via phosphorylation of p38. Stress-induced chondrocyte apoptosis decreased due to inhibition of p38 MAPK activation. In contrast, the phosphorylation of p38 MAPK increased in OA chondrocytes. Our results show that down-regulation of p38 MAPK activation inhibits chondrocyte death induced by heat stress or mechanical stress.
\end{abstract}

Correspondence to: Dr Takayuki Nishiyama, Department of Orthopaedic Surgery, Kobe University Graduate School of Medicine, 7-5-2 Kusunoki-cho, Chuo-ku, Kobe, Hyogo 670-0017, Japan

E-mail: nishiyam@med.kobe-u.ac.jp

Key words: chondrocyte, apoptosis, p38 mitogen-activated protein kinase, heat stress, mechanical stress, osteoarthritis

\section{Introduction}

Osteoarthritis (OA) is the most common degenerative disease of the human articular cartilage, especially in the population aged $\geq 65$ years (1). In the US, approximately $37 \%$ of the population has OA demonstrable by X-ray examination (2). $\mathrm{OA}$ is characterized by extracellular matrix damage and an important loss in tissue cellularity (3). Chondrocyte apoptosis, a type of programmed cell death, is an important component of the pathogenesis of OA cartilage degradation (4).

The mitogen-activated protein kinase (MAPK) pathways are major signal pathways from the cell surface to the nucleus. These signaling cascades control complex programs, such as embryogenesis, differentiation, proliferation and cell death $(5,6)$. There are three major classes of MAPKs in mammals, the extracellular signal-regulated kinases (ERKs), the c-jun $\mathrm{N}$-terminal kinase (JNK) and p38 (7). ERKs are activated by mitogens and growth factors, while JNK and p38 MAPK are activated in response to the inflammatory cytokines, TNF $\alpha$ and IL-1, as well as by cellular stress, such as heat stress, osmotic stress, reactive oxygen metabolites, and ultraviolet irradiation (8-13).

Activation of p38 MAPK results in cancer cell apoptosis initiated by retinoids, cisplatin and other chemotherapeutic agents (14). Mechanical stretch in the human saphenous vein induces activation of p38 MAPK, which is associated with apoptosis (15). Phosphorylation of p38 MAPK induced by oxidative stress has been linked to the activation of caspase 9-mediated apoptotic pathway in dopaminergic neurons (16). A p38 MAPK inhibitor was shown to decrease the number of apoptotic cells and to prevent delayed progressive degeneration in the injured area of oligodendrocytes (17). Moreover, Pelletier et al reported that chondrocyte death in experimental osteoarthritis is mediated by the p38 MAPK pathway (18). The p38 MAPK signal transduction pathway has also been implicated as a critical factor in NO-induced rabbit articular chondrocyte apoptosis (19).

Therefore, we speculated that p38 MAPK might play an important role in stress-induced apoptosis of articular cartilage, and that down-regulation of p38 MAPK might contribute to a decrease in chondrocyte apoptosis. In this study, we investigated the function of 338 MAPK in chondrocytes after heat stress or mechanical stress. 


\section{Materials and methods}

Isolation and culture of chondrocytes. Cartilage tissue samples were obtained from five patients with OA undergoing total knee replacement surgery. Diagnosis of OA was based on clinical, laboratory, and radiographic evaluations. Normal cartilage tissue samples were obtained from five age-matched patients undergoing surgery for femoral neck fracture. These five patients had no history of joint disease and had macroscopically normal cartilage. All samples were obtained in accordance with the World Medical Association Declaration of Helsinki Ethical Principles for Medical Research Involving Human Subjects. The femoral condyles and the femoral head were fixed in $4 \%$ paraformaldehyde for $24 \mathrm{~h}$, dehydrated in graded alcohol solutions, and embedded in paraffin wax. Chondrocytes were isolated from cartilage tissue and cultured. Tissue samples were minced and digested in Dulbecco's modified Eagle's medium (DMEM; Gibco BRL, Grand Island, NY) containing $0.2 \%$ collagenase (Sigma, St. Louis, MO) at $37^{\circ} \mathrm{C}$ for $2 \mathrm{~h}$. Dissociated cells were cultured in DMEM supplemented with $10 \%$ fetal bovine serum (FBS; BioWhittaker, Walkersville, $\mathrm{MD}$ ) and $100 \mathrm{U} / \mathrm{ml}$ of penicillin-streptomycin. After overnight culture, non-adherent cells were removed, and adherent cells were further incubated in fresh medium. All experiments were conducted using first-passage cells. We confirmed that all samples synthesized type II collagen, and that OA chondrocytes synthesized type $\mathrm{X}$ collagen analyzed by reverse transcription polymerase chain reaction (RT-PCR) (data not shown).

RT-PCR analysis of chondrocytes. Chondrocytes were cultured in 6-well plates with various stimulants, and RNA was extracted with the QIA-shredder and the RNeasy mini kit, according to the recommendations of the manufacturer (Qiagen, Hilden, Germany). Total RNA $(1 \mu \mathrm{g})$ was reverse-transcribed to firststrand complementary DNA with $1.25 \mu \mathrm{M}$ oligo (dT) primer in $40 \mu \mathrm{l}$ of PCR buffer II containing $2.5 \mathrm{mM} \mathrm{MgCl}_{2}, 0.5 \mathrm{mM}$ dNTP mixture, 0.5 units RNase inhibitor, and 1.25 units murine leukemia virus RT (Perkin-Elmer, Foster City, CA) at $42^{\circ} \mathrm{C}$ for $60 \mathrm{~min}$. The PCR buffer contained $1.5 \mathrm{mM} \mathrm{MgCl}{ }_{2}$, $0.2 \mathrm{mM}$ dNTP mixture, $0.5 \mu \mathrm{M}$ sense and antisense primers, 1.5 units of AmpliTaq Gold DNA polymerase, and $1 \mu \mathrm{l}$ of RT reaction mixture in $20 \mu \mathrm{l}$ of PCR buffer II (Perkin-Elmer). Thermal cycling conditions for $\mathrm{p} 38$ consisted of 40 cycles of denaturation at $95^{\circ} \mathrm{C}$ for $45 \mathrm{sec}$, annealing at $65^{\circ} \mathrm{C}$ for $45 \mathrm{sec}$, and extension at $72^{\circ} \mathrm{C}$ for $2 \mathrm{~min}$. The primers used were p38 sense, 5'-CGAAATGACCGGCTACGTGG-3', and antisense, 5'-CACTTCATCGTAGGTCAGGC-3'.

Cell fractionation. Chondrocytes were washed three times with phosphate buffered saline and lysed in MOPS buffer $(25 \mathrm{mM}$ Tris, $1 \%$ Nonidet P-40, $150 \mathrm{mM} \mathrm{NaCl}, 1.5 \mathrm{mM}$ EGTA) supplemented with a protease and phosphatase inhibitor mixture (Roche Diagnostics, Basel, Switzerland) on ice for $20 \mathrm{~min}$. The lysates were centrifuged at 15,000 revolutions per minute for $20 \mathrm{~min}$ to remove cellular debris, and the supernatants were collected, followed by the addition of $3 \mathrm{X}$ electrophoresis sample buffer (Bio-Rad, Hercules, CA) was added (20) .

Immunohistochemistry. For immunohistochemical analysis of phosphorylated $\mathrm{p} 38$, deparaffinized sections were digested with proteinase (Dako, Glostrup, Denmark) for $10 \mathrm{~min}$ and treated with $3 \%$ hydrogen peroxide (Wako Pure Chemical Industries, Osaka, Japan) to block endogenous peroxidase activity. The sections were treated in a 1:100 dilution of anti-human phosphorylated p38 antibodies (Cell Signaling Technology, Beverly, MA) at $4^{\circ} \mathrm{C}$ overnight and subsequently treated with peroxidase-labeled anti-rabbit immunoglobulin (Histofine Simple Stain MAX PO (R); Nichirei Bioscience, Tokyo, Japan) at room temperature for $30 \mathrm{~min}$. The signal was developed as a brown reaction product using peroxidase substrate 3,3'-diaminobenzidine (Histofine Simple Stain DAB Solution, Nichirei Bioscience), and the sections were examined microscopically.

Western blot analysis. The concentration of proteins was quantified by the Bradford method with the protein assay reagent (Bio-Rad). The proteins were diluted to an equal concentration with MOPS buffer. Each sample was mixed with $3 \mathrm{X}$ electrophoresis sample buffer and electrophoresed on a $7.5-15 \%$ polyacrylamide gradient gel (Biocraft, Tokyo, Japan) and transblotted electrically onto a blotting membrane (GE Healthcare, Buckinghamshire, UK). Phosphorylation of p38 was detected using an anti-human p38 monoclonal antibody (Cell Signaling Technology) and a horseradish peroxidase (HRP)-conjugated goat anti-rabbit IgG antibody (GE Healthcare), and was visualized with ECL Plus reagent (GE Healthcare) with the Chemilumino analyzer LAS-3000 mini (Fujifilm, Tokyo, Japan).

Apoptosis is known to be associated downstream with the sequential activation of caspases. Apoptosis via the p53 pathway is related to the mitochondrial pathway. Apoptotic signals were confirmed by detection of cleaved caspase 9 . Cytochrome $\mathrm{c}$ released from mitochondria associates with procaspase 9/Apaf-1, and this complex processes procaspase 9 into large and small fragments by self-cleavage under apoptotic stimulation (21). The expression of cleaved caspase 9 indicates the activation of the mitochondrial apoptotic pathway. The expression of cleaved caspase 9 was detected using mouse anti-human caspase $9 \mathrm{mAb}$ (Upstate Biotechnology, Temecula, CA) and conjugated sheep anti-mouse IgG antibody. Protein expression was determined by semi-quantification of digitally captured image using the public domain NIH Image program (National Institutes of Health, Bethesda, MD; http://rsb.info. nih.gov/nih-image/). Values were normalized to $\alpha$-tubulin expression.

Culture of normal human knee chondrocytes and exposure to heat stress or mechanical stress. Normal human knee chondrocytes (Cambrex, Charles City, IA) were cultured in a humidified atmosphere of $5 \% \mathrm{CO}_{2}$ and $95 \%$ air at $37^{\circ} \mathrm{C}$ in a Bullet kit. Before performing the experiments, we confirmed that normal human knee chondrocytes expressed type II collagen and sulfated proteoglycans but not type X collagen. Cells were grown to a subconfluent state and were then plated onto 6-well plates at a density of $3 \times 10^{5}$ cells per well in DMEM supplemented with $10 \%$ FBS and $100 \mathrm{U} / \mathrm{ml}$ of penicillinstreptomycin. The cultured cells underwent heat stress in an incubator (humidified atmosphere of $5 \% \mathrm{CO}_{2}$ and $95 \%$ air at $39^{\circ} \mathrm{C}$ for $1 \mathrm{~h}, 3 \mathrm{~h}$, and $5 \mathrm{~h}$ ). Cells were grown to a subconfluent state and were then plated onto type I collagen-coated BioFlex 
A

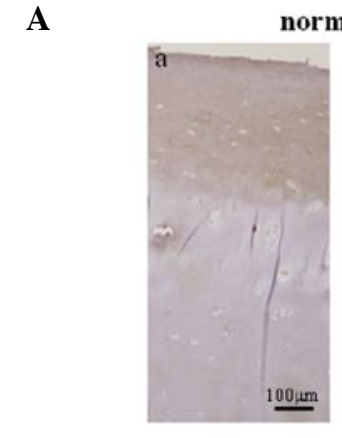

normal cartilage
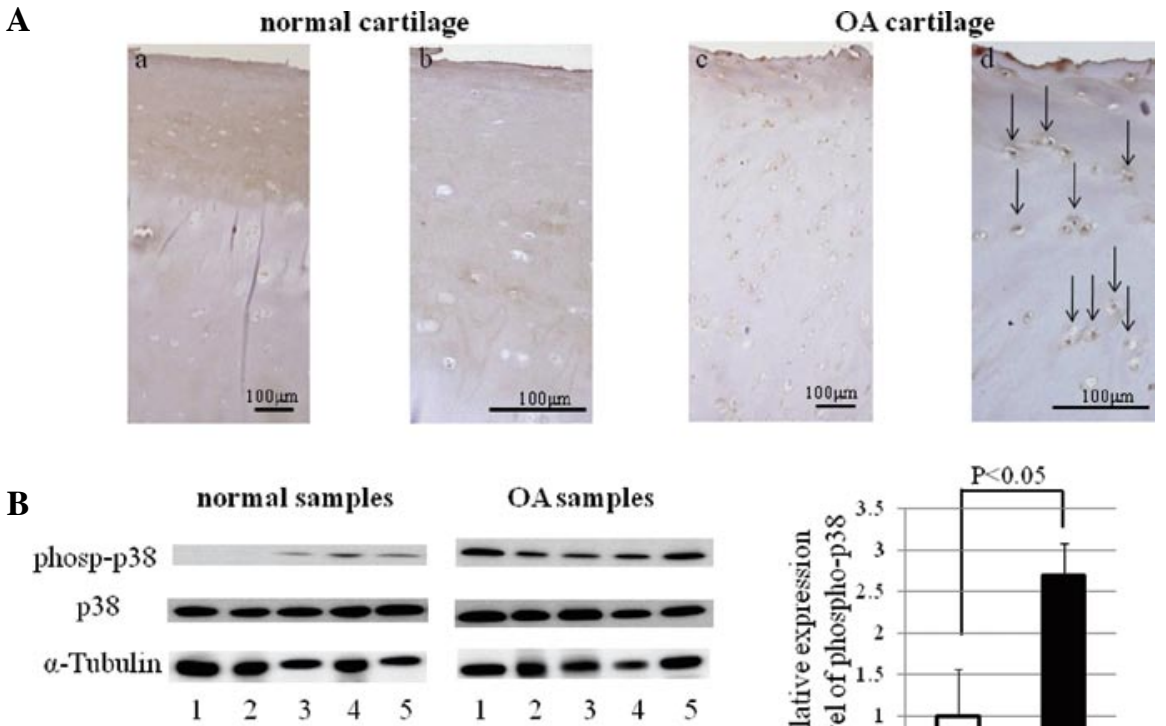

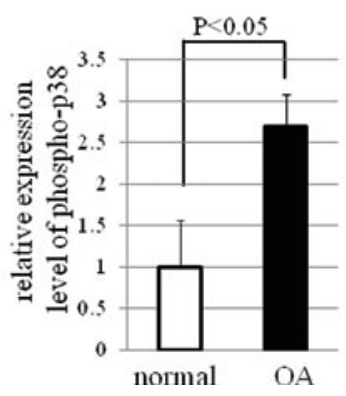

Figure 1. (A) Expression of phosphorylated p38 in osteoarthritis (OA) and normal cartilage analyzed by immunohistochemistry. The specimens were stained with anti-phosphorylated p38 antiboby. (a,b) Sections from normal cartilage. (c,d) Sections from OA cartilage. The arrows indicate stained chondrocytes. (B) Expression of p38 and phosphorylated p38 in OA and normal chondrocytes, detected by Western blotting. Ratios were obtained using the NIH Image software. Values were normalized to $\alpha$-tubulin. The bar graph shows the mean and SD of five individual samples.

plates with a deformable silicone rubber bottom surface at a density of $3 \times 10^{5}$ cells per well in DMEM/F-12 supplemented with $10 \% \mathrm{FBS}$ and $100 \mathrm{U} / \mathrm{ml}$ of penicillin-streptomycin. Cyclic stretch experiments were performed using an FX-2000 Flexercell system (Flexcell International, McKeesport, PA). Shear strain was enforced at $10 \%$ elongation for $12 \mathrm{~h}(0.25 \mathrm{~Hz})$.

Pretreatment of chondrocytes with SB203580 prior to heat stress or mechanical stress induction. SB203580 (Sigma, St. Louis, MO) is a specific inhibitor of p38 MAPK (22). Chondrocytes were pre-treated with $10 \mu \mathrm{M} \mathrm{SB} 203580$ for $1 \mathrm{~h}$ in DMEM medium supplemented with $10 \%$ FBS before the induction of heat stress or mechanical stress.

Small interfering RNA (siRNA) transfection. Lipofectamine 2000 was used to transfect p38 siRNA and non-specific siRNA control into normal human knee chondrocyte monolayers, according to the recommendations of the manufacturer (Invitrogen, San Diego, CA). Briefly, 1 day before transfection, cells were plated on a 6 -well plate in growth medium without antibiotics so that they would be $30-50 \%$ confluent at the time of transfection. Then, 100 pmoles of siRNA and Lipofectamine 2000 complexes were prepared and added to each well. After $6 \mathrm{~h}$ of transfection, the complexes were removed and fresh medium containing 10\% FBS was added. After $48 \mathrm{~h}$ of transfection, the cells were placed onto a 6 -well plate at a density of $3 \times 10^{5}$ cells per well, and heat stress or mechanical stress was enforced. Transfection efficiency was determined by RT-PCR and Western blotting.

TUNEL staining. Before induction of heat stress or mechanical stress, $3 \times 10^{5}$ chondrocytes were cultured in 6-well plates. After exposure to heat stress or mechanical stress, the chondrocytes cultured on the plates were fixed with $4 \%$ neutral buffered formalin for $10 \mathrm{~min}$, and apoptotic cells were determined using a TUNEL assay kit, according to the recommendations of the manufacturer (Wako, Osaka, Japan).

Statistical analysis. Data are expressed as the mean \pm SD. For normally distributed data, the Student's two-tailed t-test was used for comparisons between the 2 groups. $\mathrm{P}<0.05$ were considered significant.

\section{Results}

Phosphorylation of p38 MAPK is elevated in OA cartilage. Immunohistochemisty showed that phosphorylated p38 was expressed at the superficial zone in OA cartilage. However, phosphorylated p38 MAPK was not expressed at the superficial zone in normal cartilage (Fig. 1A). Phosphorylated p38 MAPK was expressed at the hypertrophic zone in both OA and normal cartilage (Fig. 1A).

Phosphorylation of p38 MAPK is elevated in OA chondrocytes. Western blotting showed that $\mathrm{p} 38$ protein was expressed in OA and normal chondrocytes. However, the expression of phosphorylated p38 was significantly higher in OA chondrocytes compared to normal chondrocytes (Fig. 1B).

Heat stress or mechanical stress induced chondrocyte apoptosis. The percentage of TUNEL-positive apoptotic cells significantly increased in a time-dependent manner after exposure to a $39^{\circ} \mathrm{C}$ heat stress (Fig. 2A). Western blotting showed that phosphorylation of p38 increased after exposure to a $39^{\circ} \mathrm{C}$ heat stress for 1,3 , or $5 \mathrm{~h}$ (Fig. 2B). Expression of cleaved caspase 9 increased in a time-dependent manner 
A

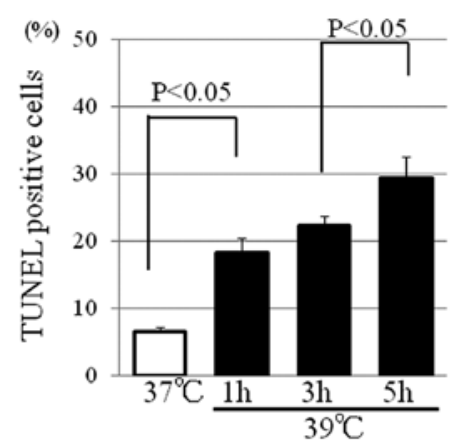

C

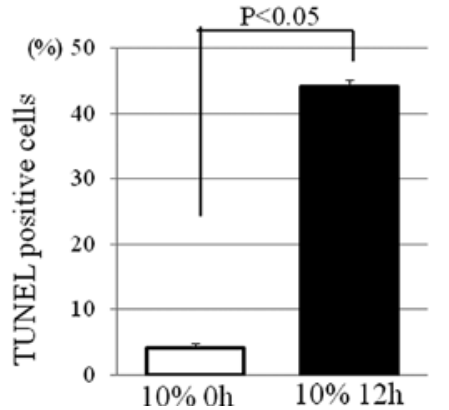

B

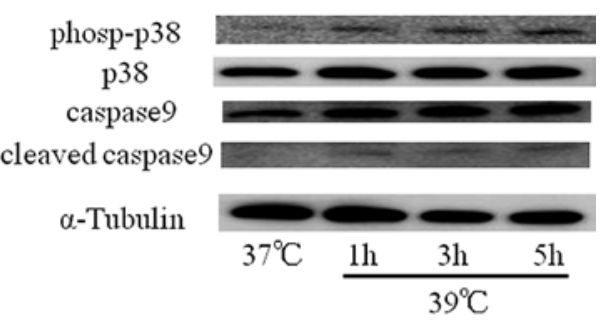

D

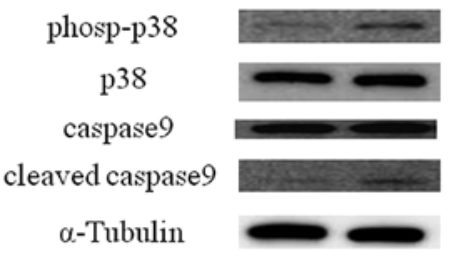

$10 \%$ oh $10 \% 12 \mathrm{~h}$

Figure 2. Effects of exposure of normal human knee chondrocytes to a $39^{\circ} \mathrm{C}$ heat stress for 1,3 , or $5 \mathrm{~h}$ or to $10 \%$ mechanical stress for $12 \mathrm{~h}$. (A) The white bar shows the percentage of TUNEL-positive apoptotic cells after exposure to $37^{\circ} \mathrm{C}$ (regular culture conditions). The black bars show the percentage of TUNELpositive apoptotic cells after exposure to a $39^{\circ} \mathrm{C}$ heat stress. At least 300 cells were counted by an observer blinded to the duration of heat stress. Bars show the mean and SD of four individual samples. (B) Western blotting of the expression of p38, phosphorylated p38, and cleaved caspase 9 after exposure to a $39^{\circ} \mathrm{C}$ heat stress. (C) Percentage of TUNEL-positive apoptotic cells after exposure to $10 \%$ mechanical stress. At least 300 cells were counted by an observer blinded to the duration of mechanical stress. Bars show the mean and SD of four individual samples. (D) Western blotting of the expression of p38, phosphorylated $\mathrm{p} 38$, and of cleaved caspase 9 after exposure to $10 \%$ mechanical stress.

A

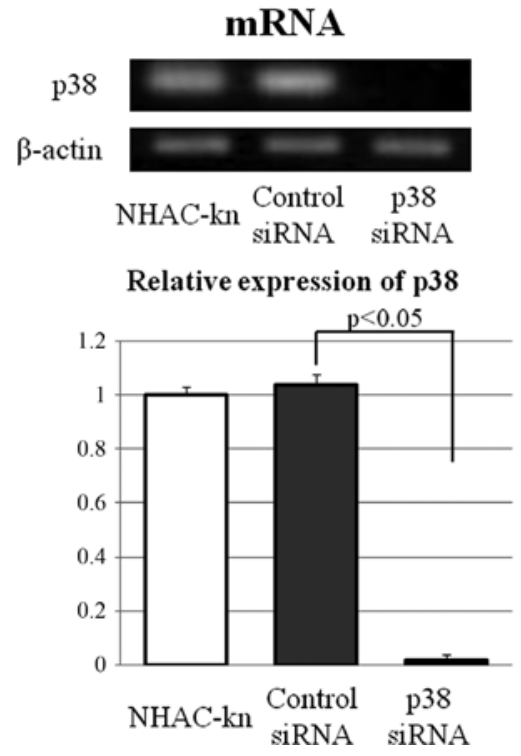

B

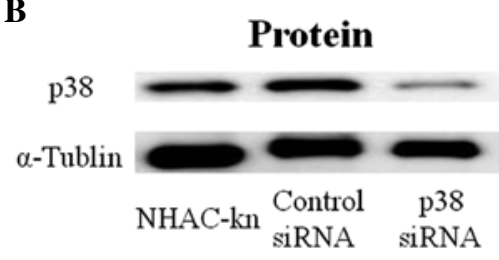

Relative expression of $\mathrm{p} 38$

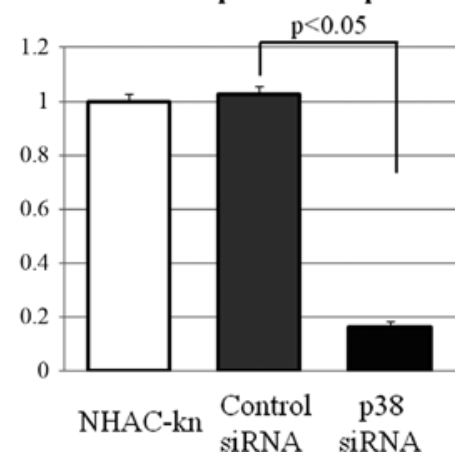

Figure 3. Efficiency of p38 small interfering RNA (siRNA) transfection in normal human knee chondrocytes (NHAC-kn). (A) Significant decrease in the expression of p38 mRNA after siRNA transfection, determined by reverse transcriptase-polymerase chain reaction. Bars show the mean and SD of four individual samples. (B) Western blot showing decreased p38 protein levels. Bars show the mean and SD.

after exposure to $39^{\circ} \mathrm{C}$ heat stress (Fig. 2B). The percentage of TUNEL-positive apoptotic cells significantly increased after exposure to $10 \%$ shear stress (Fig. 2C). Western blotting showed that phosphorylation of p38 increased after exposure to $10 \%$ shear stress for $12 \mathrm{~h}$ (Fig. 2D). Expression of cleaved caspase 9 also increased after exposure to $10 \%$ shear stress (Fig. 2D).
Down-regulation of endogenous 338 MAPK reduced heat stress or mechanical stress-induced chondrocyte apoptosis. RT-PCR showed that expression of p38 mRNA was significantly inhibited after $48 \mathrm{~h}$ of transfection with specific p38 siRNA (Fig. 3A). The expression of p38 mRNA decreased to $1.9 \%$ of the non-specific control siRNA (Fig. 3A) and that of p38 protein decreased to $16.2 \%$ of the control (Fig. 3B). 
A

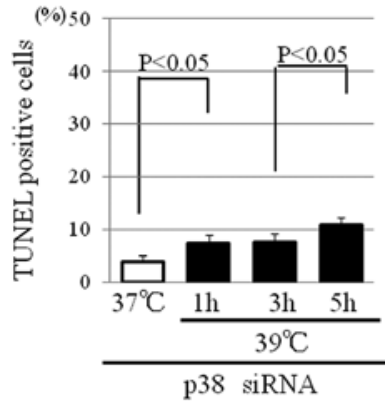

C

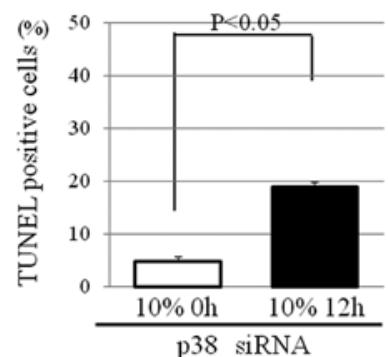

B

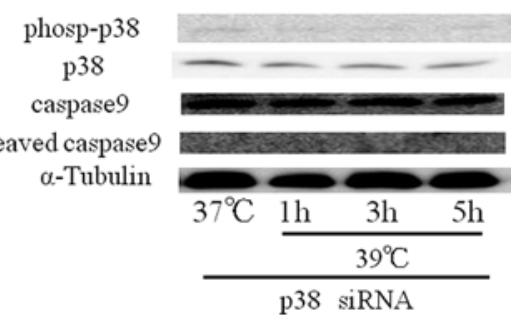

D

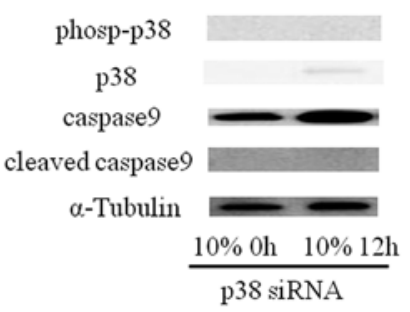

Figure 4. Effects on normal human knee chondrocytes of exposure to a $39^{\circ} \mathrm{C}$ heat stress for 1,3 , or $5 \mathrm{~h}$ or to $10 \%$ mechanical stress for $12 \mathrm{~h}$ after p 38 siRNA transfection. (A) The white bar shows the percentage of TUNEL-positive apoptotic cells under regular culture conditions after p38 siRNA transfection. The black bars show the percentage of TUNEL-positive apoptotic cells after exposure to a $39^{\circ} \mathrm{C}$ heat stress following p38 siRNA transfection. At least 300 cells were counted by an observer blinded to the duration of heat stress. The bars show the mean and SD of four individual samples. (B) Western blot analysis of the expression of $\mathrm{p} 38$, phosphorylated $\mathrm{p} 38$, and cleaved caspase 9 after exposure to a $39^{\circ} \mathrm{C}$ heat stress following $\mathrm{p} 38$ siRNA transfection. (C) Percentage of TUNEL-positive apoptotic cells after exposure to $10 \%$ mechanical stress. At least 300 cells were counted by an observer blinded to the duration of mechanical stress. Bars show the mean and SD of four individual samples. (D) Western blot analysis of the expression of p38, phosphorylated p38, and cleaved caspase 9 after exposure to $10 \%$ mechanical stress following p 38 siRNA transfection.

A

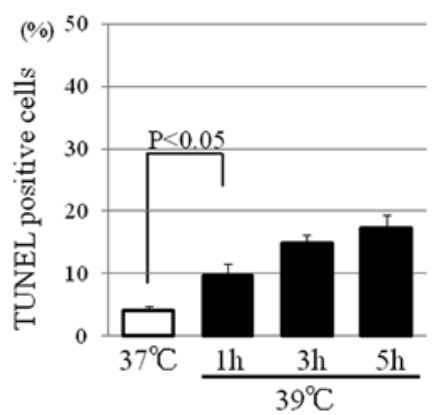

C

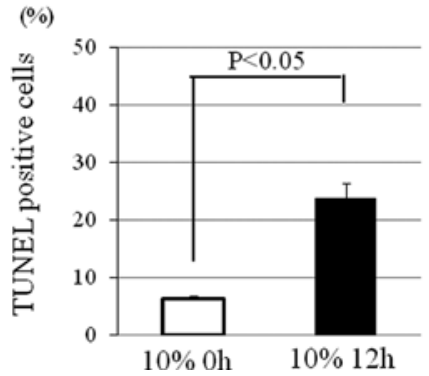

B

phosp-p38

p38

caspase 9

cleaved caspase 9

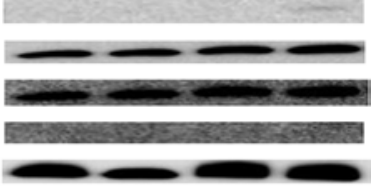

$37^{\circ} \mathrm{C} \frac{1 \mathrm{~h} \quad 3 \mathrm{~h} \quad 5 \mathrm{~h}}{39^{\circ} \mathrm{C}}$

D

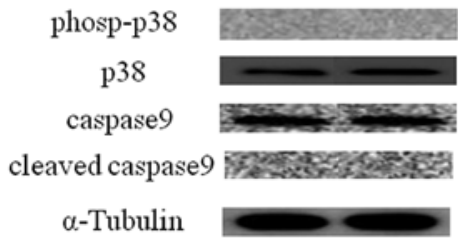

$10 \%$ oh $10 \% 12 \mathrm{~h}$

Figure 5. Effects of exposure to a $39^{\circ} \mathrm{C}$ heat stress for 1,3 , or $5 \mathrm{~h}$ or to $10 \%$ mechanical stress for $12 \mathrm{~h}$ on normal human knee chondrocytes pre-treated with SB203580 $(10 \mu \mathrm{M})$. (A) The white bar shows the percentage of TUNEL-positive apoptotic cells under regular culture conditions after treatment with SB203580. The black bars show the percentage of TUNEL-positive apoptotic cells after exposure to $39^{\circ} \mathrm{C}$ heat stress following treatment with SB203580. At least 300 cells were counted by an observer blinded to the duration of heat stress. Bars show the mean and SD of four individual samples. (B) Western blot analysis of expression of p38, phosphorylated p38, and cleaved caspase 9 after exposure to a $39^{\circ} \mathrm{C}$ heat stress following treatment with SB203580. (C) Percentage of TUNEL-positive apoptotic cells after exposure to $10 \%$ mechanical stress following treatment with SB203580. At least 300 cells were counted by an observer blinded to the duration of mechanical stress. Bars show the mean and SD of four individual samples. (D) Western blot analysis of the expression of p38, phosphorylated p38, and cleaved caspase 9 after exposure to $10 \%$ mechanical stress following treatment with SB203580.

The percentage of TUNEL-positive apoptotic cells was increased by heat stress. However, the number of apoptotic cells induced by heat stress after p38 siRNA transfection (Fig. 4A) was significantly lower in comparison with that of apoptotic cells when p38 siRNA was not transfected. Western blotting confirmed that expressions of $\mathrm{p} 38$ and phosphorylated $\mathrm{p} 38$ 
MAPK were inhibited with p38 siRNA transfection (Fig. 4B). Cleaved caspase 9 expression was not detected after p38 siRNA transfection (Fig. 4B). Similarly, the percentage of TUNEL-positive apoptotic cells increased by $10 \%$ shear stress. However, the number of apoptotic cells induced by shear stress was significantly inhibited when p38 siRNA was transfected in comparison with that of apoptotic cells when p38 siRNA was not transfected (Fig. 4C). Western blotting confirmed that phosphorylated p38 expression was inhibited with p38 siRNA transfection (Fig. 4D). Cleaved caspase 9 expression was also decreased with p38 siRNA transfection (Fig. 4D).

Inhibition of $p 38$ activity reduced heat stress or mechanical stress-induced chondrocyte apoptosis. The percentage of TUNEL-positive apoptotic cells was decreased when chondrocytes were pre-treated with SB203580 for $1 \mathrm{~h}$ before exposure to $39^{\circ} \mathrm{C}$ heat stress (Fig. 5A). Western blotting showed that the expressions of phosphorylated p38 and of cleaved caspase 9 were decreased when chondrocytes were pre-treated with $10 \mu \mathrm{M}$ SB203580 (Fig. 5B). The percentage of TUNEL-positive apoptotic cells was decreased when chondrocytes were pre-treated with SB203580 for $1 \mathrm{~h}$ before exposure to $10 \%$ shear stress for $12 \mathrm{~h}$ (Fig. 5C). Western blotting showed that phosphorylation of p38 and expression of cleaved caspase 9 decreased compared to that in untreated samples when chondrocytes were pre-treated with $10 \mu \mathrm{M}$ SB203580 (Fig. 5D).

\section{Discussion}

Cartilage cells are mainly responsible for the anabolic-catabolic balance required for matrix maintenance and tissue function (23). Chondrocytes in OA receive physical stresses such as unphysiological high-weight loading and high temperature (24). Various biological and chemical stress factors are believed to be involved in the onset and progression of the pathogenesis of OA (25).

In patients with OA, the intra-articular temperature is possibly elevated to a higher degree due to local inflammation and aberrant frictional force induced by non-physiologial mechanical loading (26). Mitrovic et al reported that hyperthermia induced damage to articular cartilage (27). Ye et al showed that heat stress induced articular chondrocyte apoptosis in a rat model (28). It was reported that physiological movement of a joint elevates the temperature in the joint cavity by $2.5^{\circ} \mathrm{C}(26)$. Hojo et al reported that appropriate heat stimulation positively affects cell viability and the proteoglycan metabolism of articular cartilage, whereas too much heat stimulation produces negative effects (29). However, the mechanism of signal transduction of heat stress remains unclear in articular chondrocytes.

Previous studies have shown that intermittent hydrostatic pressure induces apoptosis in vitro in a load- and a timedependent manner and is characterized by a loss of chondrocyte viability, internucleosomal DNA fragmentation, and activation of caspases (30). Furthermore, optimal mechanical stress stimulation was shown to maintain the structure and function of articular cartilage, whereas excessive mechanical force was shown to lead to a loss of cartilage and the onset of OA (31-33). In addition, we have previously demonstrated that excessive shear stress induces chodrocyte apoptosis via the p53 pathway (34).

In this study, we have demonstrated that heat stress or mechanical stress increases the phophorylation of p38 MAPK, and induces chondrocyte apoptosis via the caspase 9 pathway. Furthermore, we have shown that down-regulation of $\mathrm{p} 38$ MAPK expression by specific p38 MAPK siRNA transfection decreased chondrocyte apoptosis in response to both heat stress and mechanical stress. Our findings indicate that p38 MAPK plays a critical function in chondrocyte apoptosis induced by heat stress or mechanical stress.

Under physiological conditions, OA cartilage has a higher number of apoptotic chondrocytes compard to that of normal cartilage (4). Hashimoto et al reported that chondrocyte apoptotic cells are found in the superficial and middle zone of OA cartilage (35). In this study, we have demonstrated that phosphorylated p38 MAPK was expressed in the superficial and middle zone in OA cartilage, but not in normal cartilage. Furthermore, OA chondrocytes expressed phosphorylated p 38 MAPK protein more than normal chondrocytes. These results suggest that chondrocyte apoptosis in response to stress is closely linked to activation of $\mathrm{p} 38$ MAPK.

We also found that phosphorylated p38 MAPK was expressed in the deep zone (hypertrophic zone) of both OA and normal cartilage. This phenomenon is explained by previous studies showing that hypertrophic chondrocytes direct the formation of the mineralized matrix, attract blood vessels, and undergo apoptosis (36-38).

Finally, we demonstrated that inhibition of p38 MAPK activity by SB203580 reduced chondrocyte apoptosis in response to heat stress. This finding supports the notion that SB203580 could be used as a drug for OA therapy.

In conclusion, we have demonstrated that heat stress or mechanical stress increases chondrocyte apoptosis via phosphorylation of $\mathrm{p} 38$. Stress-induced chondrocyte apoptosis was decreased due to inhibition of p38 MAPK activation when chondrocytes were pre-treated with SB203580 or transfected with $\mathrm{p} 38$ specific siRNA. The phosphorylation of p38 MAPK was increased in OA chondrocytes. Based on our results, down-regulation of p38 MAPK activation inhibits chondrocyte death induced by heat stress. Therefore, evaluation of the interaction between phosphorylation of $\mathrm{p} 38$ and chondrocyte apoptosis could be one of the keys to elucidate OA etiology.

\section{Acknowledgements}

We thank Ms. Kyoko Tanaka, Ms. Minako Nagata, and Ms. Maya Yasuda for technical assistance and Janina Tubby for English language assistance.

\section{References}

1. Hamerman D: Clinical implications of osteoarthritis and ageing. Ann Rheum Dis 54: 82-85, 1995.

2. Threlkeld AJ and Currier DP: Osteoarthritis. Effects on synovial joint tissues. Phys Ther 68: 364-370, 1988.

3. Vignon E, Arlot M, Meunier P and Vignon G: Quantitative histological changes in osteoarthritic hip cartilage. Morphometric analysis of 29 osteoarthritic and 26 normal human femoral heads. Clin Orthop Relat Res 103: 269-278, 1974.

4. Blanco FJ, Guitian R, Vazquez-Martul E, de Toro FJ and Galdo F: Osteoarthritis chondrocytes die by apoptosis. A possible pathway for osteoarthritis pathology. Arthritis Rheum 41: 284-289, 1998. 
5. English JM and Cobb MH: Pharmacological inhibitors of MAPK pathways. Trends Pharmacol Sci 23: 40-45, 2002.

6. Chang L and Karin M: Mammalian MAP kinase signalling cascades. Nature 410: 37-40, 2001.

7. Thalhamer T, McGrath MA and Harnett MM: MAPKs and their relevance to arthritis and inflammation. Rheumatology (Oxford) 47: 409-414, 2008

8. Derijard B, Hibi M, Wu IH, et al: JNK1: a protein kinase stimulated by UV light and Ha-Ras that binds and phosphorylates the c-Jun activation domain. Cell 76: 1025-1037, 1994.

9. Drosos AA: Newer immunosuppressive drugs: their potential role in rheumatoid arthritis therapy. Drugs 62: 891-907,2002.

10. Kyriakis JM, Banerjee P, Nikolakaki E, et al: The stress-activated protein kinase subfamily of c-Jun kinases. Nature 369: 156-160, 1994.

11. Raingeaud J, Gupta S, Rogers JS, et al: Pro-inflammatory cytokines and environmental stress cause p38 mitogen-activated protein kinase activation by dual phosphorylation on tyrosine and threonine. J Biol Chem 270: 7420-7426, 1995.

12. Verheij M, Bose R, Lin XH, et al: Requirement for ceramideinitiated SAPK/JNK signalling in stress-induced apoptosis. Nature 380: 75-79, 1996.

13. Freshney NW, Rawlinson L, Guesdon F, et al: Interleukin-1 activates a novel protein kinase cascade that results in the phosphorylation of Hsp27. Cell 78: 1039-1049, 1994.

14. Olson JM and Hallahan AR: p38 MAP kinase: a convergence point in cancer therapy. Trends Mol Med 10: 125-129, 2004.

15. Cornelissen J, Armstrong J and Holt CM: Mechanical stretch induces phosphorylation of p38-MAPK and apoptosis in human saphenous vein. Arterioscler Thromb Vasc Biol 24: 451-456, 2004.

16. Choi WS, Eom DS, Han BS, et al: Phosphorylation of p38 MAPK induced by oxidative stress is linked to activation of both caspase-8- and -9-mediated apoptotic pathways in dopaminergic neurons. J Biol Chem 279: 20451-20460, 2004.

17. Horiuchi H, Ogata T, Morino T, Chuai $\mathrm{M}$ and Yamamoto $\mathrm{H}$ Continuous intrathecal infusion of SB203580, a selective inhibitor of p38 mitogen-activated protein kinase, reduces the damage of hind-limb function after thoracic spinal cord injury in rat. Neurosci Res 47: 209-217, 2003

18. Pelletier JP, Fernandes JC, Jovanovic DV, Reboul P and MartelPelletier J: Chondrocyte death in experimental osteoarthritis is mediated by MEK $1 / 2$ and p38 pathways: role of cyclooxygenase- 2 and inducible nitric oxide synthase. J Rheumatol 28 : 2509-2519, 2001

19. Wang $\mathrm{H}$, Wang $\mathrm{Z}$, Chen $\mathrm{J}$ and $\mathrm{Wu} \mathrm{J}$ : Apoptosis induced by NO via phosphorylation of p38 MAPK that stimulates NF-kappaB p53 and caspase- 3 activation in rabbit articular chondrocytes. Cell Biol Int 31: 1027-1035, 2007.

20. Hayashi S, Miura Y, Nishiyama T, et al: Decoy receptor 3 expressed in rheumatoid synovial fibroblasts protects the cells against fas-induced apoptosis. Arthritis Rheum 56: 1067-1075, 2007.

21. Zou H, Li Y, Liu X and Wang X: An APAF-1.cytochrome c multimeric complex is a functional apoptosome that activates procaspase-9. J Biol Chem 274: 11549-11556, 1999.

22. Cuenda A, Rouse J, Doza YN, et al: SB 203580 is a specific inhibitor of a MAP kinase homologue which is stimulated by cellular stresses and interleukin-1. FEBS Lett 364: 229-233, 1995.
23. Aigner T, Soder S, Gebhard PM, McAlinden A and Haag J: Mechanisms of disease: role of chondrocytes in the pathogenesis of osteoarthritis-structure, chaos and senescence. Nat Clin Pract Rheumatol 3: 391-399, 2007.

24. Kubo T, Arai Y, Takahashi K, et al: Expression of transduced HSP70 gene protects chondrocytes from stress. J Rheumatol 28: 330-335, 2001

25. Tonomura H, Takahashi KA, Mazda O, et al: Glutamine protects articular chondrocytes from heat stress and NO-induced apoptosis with HSP70 expression. Osteoarthritis Cartilage 14: 545-553, 2006

26. Tepic S, Macirowski T and Mann RW: Experimental temperature rise in human hip joint in vitro in simulated walking. J Orthop Res 3: 516-520, 1985.

27. Mitrovic DR, Gruson M and Ryckewaert A: Local hyperthermia and cartilage breakdown: histochemical and metabolic studies on rabbit articular cartilage in vitro. J Rheumatol 8: 193-203, 1981.

28. Ye J, Haro H, Takahashi M, Kuroda H and Shinomiya K Induction of apoptosis of articular chondrocytes and suppression of articular cartilage proteoglycan synthesis by heat shock. J Orthop Sci 8: 387-395, 2003.

29. Hojo T, Fujioka M, Otsuka G, Inoue S, Kim U and Kubo T: Effect of heat stimulation on viability and proteoglycan metabolism of cultured chondrocytes: preliminary report. J Orthop Sci 8: 396-399, 2003.

30. Islam N, Haqqi TM, Jepsen KJ, et al: Hydrostatic pressure induces apoptosis in human chondrocytes from osteoarthritic cartilage through up-regulation of tumor necrosis factor-alpha, inducible nitric oxide synthase, p53, c-myc, and bax-alpha, and suppression of bcl-2. J Cell Biochem 87: 266-278, 2002.

31. Guilak F, Ratcliffe A, Lane N, Rosenwasser MP and Mow VC: Mechanical and biochemical changes in the superficial zone of articular cartilage in canine experimental osteoarthritis. J Orthop Res 12: 474-484, 1994.

32. Loening AM, James IE, Levenston ME, et al: Injurious mechanical compression of bovine articular cartilage induces chondrocyte apoptosis. Arch Biochem Biophys 381: 205-212, 2000.

33. Grodzinsky AJ, Levenston ME, Jin M and Frank EH: Cartilage tissue remodeling in response to mechanical forces. Annu Rev Biomed Eng 2: 691-713, 2000.

34. Hashimoto S, Nishiyama T, Hayashi S, et al: Role of p53 in human chondrocyte apoptosis in response to shear strain. Arthritis Rheum 60: 2340-2349, 2009.

35. Hashimoto S, Ochs RL, Komiya S and Lotz M: Linkage of chondrocyte apoptosis and cartilage degradation in human osteoarthritis. Arthritis Rheum 41: 1632-1638, 1998.

36. Kronenberg HM: Developmental regulation of the growth plate. Nature 423: 332-336, 2003

37. Hatori M, Klatte KJ, Teixeira CC and Shapiro IM: End labeling studies of fragmented DNA in the avian growth plate: evidence of apoptosis in terminally differentiated chondrocytes. J Bone Miner Res 10: 1960-1968, 1995.

38. Gibson GJ, Kohler WJ and Schaffler MB: Chondrocyte apoptosis in endochondral ossification of chick sterna. Dev Dyn 203: 468-476, 1995. 